\title{
Adjuvant Chemotherapy with Chinese Herbal Medicine Formulas Versus Placebo in Patients with Lung Adenocarcinoma after Radical Surgery: a Multicenter, Randomized, Double-Blind, Placebo- Controlled Trial
}

Qin Wang ${ }^{1 \dagger}$, Lijing Jiao ${ }^{1,2+}$, Shengfei Wang ${ }^{3 \dagger}$, Peiqi Chen ${ }^{1}$, Ling Bi ${ }^{1}$, Di Zhou ${ }^{1}$, Jialin Yao ${ }^{1}$, Jiaqi Li ${ }^{1}$, Liyu Wang ${ }^{1}$, Zhiwei Chen ${ }^{4}$, Yingjie Jia ${ }^{5}$, Ziwen Zhang ${ }^{6}$, Weisheng Shen ${ }^{7}$, Weirong Zhu ${ }^{8}$, Jianfang X ${ }^{9}$, Yong Gao ${ }^{10}$, Ling $\mathrm{Xu}^{1^{*}}$ and Yabin Gong ${ }^{1 *}$

\begin{abstract}
Background: The toxicity and side effects caused by adjuvant chemotherapy (ACT) after radical surgery for lung adenocarcinoma (LAC) lead to early termination frequently. This study was conducted to provide an objective basis for the effect of Chinese herbal medicine formulas (CHMFs) combined with chemotherapy in reducing toxicity and enhancing efficacy of ACT.
\end{abstract}

Method: From February 17th, 2012 to March 20th, 2015, 233 patients from 7 hospitals diagnosed with LAC in IB IIIA stage were randomly assigned into ACT + CHMF group (116 patients) and ACT + placebo group (117 patients). CHMF was taken orally until the end of chemotherapy. Chemotherapy-related toxic, side effects were investigated as the primary outcome. Disease-free survival (DFS) and overall survival (OS) were used as the secondary outcome.

Results: At one week following chemotherapy, the incidence of dry mouth, diarrhea and thrombocytopenia significantly decreased in CHMF group $(P=0.017, P=0.033, P=0.019$, respectively). At two weeks following chemotherapy, fatigue and diarrhea were more obvious in the placebo group $(P=0.028, P=0.025$, respectively). In addition, patients in the CHMF group showed an increase in median DFS from 37.1 to 51.5 months compared with placebo group although there was no statistical significance $(P=0.16)$. In the stage IB subgroup, the CHMF group had a significantly better DFS (HR $(95 \% \mathrm{Cl})=0.53(0.28-0.99), P=0.046)$. There was no significant difference in OS between the groups $(P=0.72)$.

Conclusion: For patients with $L A C, A C T$ combined with CHMF after radical surgery can prolong the DFS time especially in the early stage, and reduces the chemotherapy-related toxic and side effects.

(Continued on next page)

\footnotetext{
*Correspondence: xulq67@aliyun.com; gongyabin@hotmail.com

${ }^{\dagger}$ Qin Wang, Lijing Jiao and Shengfei Wang contributed equally to this work.

${ }^{1}$ Department of Oncology, Yueyang Hospital of Integrated Traditional

Chinese and Western Medicine, Shanghai University of Traditional Chinese

Medicine, Shanghai, China

Full list of author information is available at the end of the article
}

(c) The Author(s). 2020 Open Access This article is distributed under the terms of the Creative Commons Attribution 4.0 International License (http://creativecommons.org/licenses/by/4.0/), which permits unrestricted use, distribution, and reproduction in any medium, provided you give appropriate credit to the original author(s) and the source, provide a link to the Creative Commons license, and indicate if changes were made. The Creative Commons Public Domain Dedication waiver (http://creativecommons.org/publicdomain/zero/1.0/) applies to the data made available in this article, unless otherwise stated. 
(Continued from previous page)

Trial Registration: NCT 01441752. Registered 14 July, 2011.

Keywords: Lung adenocarcinoma, Chinese herbal medicine formulas, Adjuvant chemotherapy, Adverse events, Disease-free survival

\section{Background}

Lung cancer is one of the most common causes of cancerrelated death all over the world. Non-small cell lung cancers (NSCLC) account for $80 \%$ of lung malignancies, of these, roughly $50 \%$ are adenocarcinoma, and $18 \%$ of Lung adenocarcinoma have an overall 5-year survival [1]. Radical surgery combined with systematic lymphadenectomy is regarded as the primary treatment modality for early stage NSCLC. But even after complete R0 resection, there is still high risk of local recurrence or distant metastases. According to the 8th edition of TNM classification for lung cancer, the 5-year survival rate after surgery is disappointingly low, ranging from 58 to $73 \%$ in patients with stage I tumors, 36 to $46 \%$ in those with stage II tumors, and only 19 to $24 \%$ in those with stage IIIA tumors [2].

In a meta-analysis including 5 largest independent clinical trials, the Lung Adjuvant Cisplatin Evaluation (LACE) collaborative group demonstrated that postoperative cisplatin-based chemotherapy significantly improved survival in patients with NSCLC [3]. The 5-year survival rate was $5.4 \%$ higher in the cisplatin-based ACT group than in the group receiving no ACT. Another trial by Adjuvant Navelbine International Trialist Association (ANITA) showed the survival benefit of adjuvant vinorelbine plus cisplatin (NP) versus control in patients with completely resected stage IB IIIA NSCLC. After a median follow-up of 76 months, median survival was 65.7 and 43.7 months in the chemotherapy group and the observation group respectively [4]. Based on results from these studies and relevant analyses, the guidelines issued by American Society of Clinical Oncology (ASCO) and U.S. National Comprehensive Cancer Network (NCCN) recommend that cisplatin-based two drugs ACT be given to post-surgical NSCLC patients suffering from resectable stage II IIIA NSCLC or stage IB NSCLC with high risk factors $[5,6]$.

Although ACT improves the survival of NSCLC patients after surgical resection, it is undeniable that there is a possibility of potential complications or fatal adverse events. In a trial by ANITA, febrile neutropenia occurred in $9 \%$ of the patients, with $2 \%$ resulting in death [4]. In the JBR.10 trial, hematological toxicity led to death in 2 patients [7]. In a pooled analysis of five trials, the overall incidence of grade 4 toxicity was $32 \%$ in the chemotherapy group, and the main reasons for receiving less than the planned number of cycles were patient refusal (35\%) and toxicity (34\%) [3].
In China, traditional Chinese medicine (TCM) is mainly used clinically in combination with ACT to cope with chemotherapy-related side effects. In addition, TCM used alone in advanced NSCLC patients intolerant to chemotherapy was reported to be able to prevent recurrence and metastasis and prolong the survival time $[8,9]$. A retrospective small-sample study was performed in patients with stage II IIIA NSCLC. It was revealed that compared with the patients receiving $\mathrm{ACT}$ alone, those receiving Chinese medicine + ACT enjoyed a longer time without relapse or metastasis [10]. Our previous study showed that Chinese herbal medicines based on syndrome differentiation were able to improve the symptoms, including nausea, fatigue, pain and dry mouth, and also alleviate some chemotherapy-related side effects $[11,12]$.

The clinical therapeutic benefits of TCM still lack reliable evidence from prospective study. Therefore, this multi-center, prospective, randomized, double-blind trial was designed to demonstrate a higher benefit for $\mathrm{CHMF}+\mathrm{ACT}$.

\section{Materials and Methods \\ Study Design and Participants}

Participants meeting the following inclusion criteria were enrolled from medical centers: completely resected stage Ib IIIa NSCLC, aged 18 75 years, an Eastern Cooperative Oncology Group performance status (ECOG PS) scale of $0 \sim 2$ and adequate hematological, biochemical and organ functions. Chemotherapy was started within 6 weeks after surgery and consisted of 4 cycles of treatment with cisplatin/carboplatin and vinorelbine. Any patient meeting the following exclusion criteria was excluded from this clinical study: having other primary malignant tumors; incomplete resection or uncertain to have resection; serious problems of the heart, liver or kidney with severe dysfunction; being pregnant or breast-feeding; having mental or cognitive disorders that would influence judgment of quality of life (QoL) in this study; receiving or about to receive chemotherapy; being participating in other drug trials; allergic to the drug in the study. The study protocol was approved by the institutional review boards (IRBs) of the participating medical centers, the independent National Ethics Committee and Chinese Medicinal Agency, and was registered at Clinical Trials. gov (number: NCT01441752; date: July 14, 2011). All of the participants provided written informed consent before enrolment. 


\section{Procedure}

Eligible patients were randomly assigned 1:1 to receive ACT with CHMF or with placebo within 6 weeks after surgery. Central randomization was performed by a clinical research organization (CRO) (Shanghai Clinical Research Center, Shanghai, China; http://www.scrcnet. org). Random numbers were automatically generated by computer referring to pre-configured stratified factors [stage (Ib vs IIa vs IIb vs IIIa), gender (male, female) and the medical center] before administration of ACT. Then the CRO provided the random number to each participating center. The block size and treatmentassignment table were not available to the researchers until the end of the study.

\section{Syndrome Differentiation Criteria}

The CHMFs were prescribed based on syndrome differentiation. Syndrome differentiation criteria were based on "The Guiding Principles of Clinical Research of New Chinese Medicine (trial)" (China Pharmaceutical Technology Publishing House, 2002) and "Shanghai diagnosis and treatment routine of TCM Syndrome"(Shanghai Municipal Health Bureau edit). Three syndromes of TCM are set as follows:

Qi deficiency syndrome is composed of the following symptoms: cough with phlegm, poor appetite, lassitude and weakness, pale and plump tongue; secondary symptoms of spontaneous sweating, loose stool or soft slippery pulse.

Yin deficiency syndrome contains main symptoms of cough with scanty phlegm, dry mouth, reddish tongue; secondary symptoms of night sweating, heartburn and insomnia, low fever, thread and rapid pulse.

Qi and Yin deficiency syndrome contains main symptoms of cough with scanty phlegm, shortness of breath, lassitude and weakness, thirst without the desire to drink; secondary symptoms of spontaneous sweating, night sweating, reddish tongue or tongue with teeth marks, and thread and weak pulse.

The diagnosis can be given with at least two of the main symptoms and one of the secondary symptoms. Each formula was prescribed by one professor who had worked in Longhua hospital for more than 50 years to facilitate clinical research.

Patients who diagnosed with Qi syndrome deficiency, Yin syndrome deficiency received treatment with basic herbs adding YiQi formula and YangYin formula respectively. Patients with both Qi and Yin syndrome deficiency prescribed with basic herbs adding the combination of YiQi and YangYin formula granules. In placebo group, patients have the corresponding placebo granules. The composition of the formulas was shown in Table 1.

\section{Pharmaceutical Ingredients}

Three formulas (batch numbers: 1106070, 1106011, 1106041) were dynamic cycle extracted, then concentrated and spray-dried to make granules by Jiangyin Tianjiang Pharmaceutical Co. Ltd. (Jiangsu, China).

In conformity with Good Clinical Practice (GCP), in double-blind clinical trials, the investigational drug and placebo should be identical in appearance (color, viscosity, hardness, and other physical properties), smell, packaging, labeling, and other characteristics. It is difficult to define a placebo in Chinese herbal medicine where allnatural substances are potentially therapeutic. Therefore, the raw materials for the placebo including $10 \%$ of CHMF, and food color, artificial flavors. Ingredient of food color and artificial flavors can be found in our previous study [12]. At this concentration, the placebo was reported having no physiological activity $[13,14]$.

\section{Intervention}

Patients received continued ACT (vinorelbine, $25 \mathrm{mg}$ / $\mathrm{m}^{2}$, on days 1 and 8 , twice every 21 days and cisplatin $75 \mathrm{mg} / \mathrm{m}^{2}$, on day 1 , once every 21 days or carboplatin AUC (area under the curve) $=5$, on day 1 , once every 21 days). Meanwhile, patients enrolled in the study received treatment with CHMFs or matched placebo twice a day until the end of chemotherapy.

The CHMF and placebo granules were administered on the first day after chemotherapy. The medicine was dissolved in $160 \mathrm{~mL}$ of warm water for drinking at 9:30 a.m. and 15:00 p.m. All procedures were carried out under the supervision of clinical research pharmacists. The processed herbs and the granules were prepared in compliance with Good Manufacturing Practice (GMP).

Chest computed tomography scan was carried out every 2 cycles to evaluate the clinical response. Within the first 2 years following the end of chemotherapy, a chest CT scan was performed every 2 months, then from the third year every 6 months [15]. Blood chemistry, hematology, concomitant medications, and adverse events were assessed on day 1 of each 21-day cycle. Adverse events including toxicity and side effects were reported according to Common Terminology Criteria for Adverse Events V3.0 (CTC AE) issued by National Cancer Institute (NCI) (http://ctep. info.nih.gov). All of the unexpected responses possibly or definitely related to the study were reported. In case of serious adverse events (SAEs), treatment was stopped immediately and appropriate treatment was provided. Patients with disease progression continued to be assessed every 3 months.

\section{Statistical Analysis}

All of the statistical analyses on data were performed using the software SPSS 18.0. Chi-square test was employed for 
Table 1 Composition of the basic herbs and added formulas with different syndromes

\begin{tabular}{|c|c|c|c|c|c|}
\hline \multicolumn{6}{|c|}{ Basic Herbs for all patients } \\
\hline $\begin{array}{l}\text { Chinese } \\
\text { name }\end{array}$ & $\begin{array}{l}\text { Chinese name } \\
\text { (Pinyin) }\end{array}$ & Pharmaceutical name & English name & $\begin{array}{l}\text { Produced } \\
\text { from }\end{array}$ & $\begin{array}{l}\text { Dosage } \\
\text { (grams) }\end{array}$ \\
\hline 夏枯草 & Xia Ku Cao & Prunlla vulgaris $\mathrm{L}$. & Spica Prunellae & Dry spikes & 15 \\
\hline 生南星 & Sheng Nan Xing & Arisaema erubescens (Wall.) Schott. & Arisaema Rhizoma Arisaematis & Dry rhizoma & 30 \\
\hline 蛇六谷 & She Liu Gu & Amorphophallus konjac K.Koch & Rhizoma Amorphophalli & Dry tubers & 30 \\
\hline 山慈菇 & Shan Ci Gu & Cremastra appendiculata (D.Don) Makino & $\begin{array}{l}\text { Pseudobulbus Cremastrae Seu } \\
\text { Pleiones }\end{array}$ & $\begin{array}{l}\text { Dry } \\
\text { pseudobulb }\end{array}$ & 15 \\
\hline 泽漆 & Ze Qi & Euphorbia helioscopia L. & Euphorbiae Helioscopiae & Herba & 15 \\
\hline 石上柏 & Shi Shang Bai & Selaginella Doederleinii Hieron & Selaginella Doederleinii & Herba & 30 \\
\hline 石见穿 & Shi Jian Chuan & Salvia chinensis Benth. & Salviae Chinensis & Herba & 30 \\
\hline 重楼 & Chong Lou & $\begin{array}{l}\text { Paris polyphylla Smith var. chinensis } \\
\text { (Franch.) H.Hara }\end{array}$ & Rhizoma Paridis & Dry rhizoma & 15 \\
\hline 海藻 & Hai Zao & Sargassum & Seaweed & Leaves & 15 \\
\hline 瓜萎皮 & Gua Lou Pi & Trichosanthes kirilowii Maxim. & Pericarpium Trichosanthis & Pericarp & 15 \\
\hline 猫爪草 & Mao Zhua Cao & Ranunculus ternatus Thunb. & Radix Ranunculus Ternati & Herba & 15 \\
\hline 生牡蚛 & Sheng Mu Li & Concha Ostreae & Oyster Shell & Shell & 30 \\
\hline 大來 & Da Zao & Ziziphus jujuba Mill. & Fructus Jujubae & Fruit & 9 \\
\hline \multicolumn{6}{|c|}{ For Qi syndrome deficiency, add Yiqi formula: } \\
\hline $\begin{array}{l}\text { Chinese } \\
\text { name }\end{array}$ & $\begin{array}{l}\text { Chinese } \\
\text { name(Pinyin) }\end{array}$ & Pharmaceutical name & English name & $\begin{array}{l}\text { Produced } \\
\text { from }\end{array}$ & $\begin{array}{l}\text { Dosage } \\
\text { (grams) }\end{array}$ \\
\hline 黄芪 & Huang Qi & Astragalus membranaceus Fisch. ex Bunge & Milkvetch Root Radix Astragali & Dry rhizoma & 30 \\
\hline 党参 & Dang Shen & Codonopsis pilosula (Franch.) Nannf. & Codonopsis Radix & Dry rhizoma & 9 \\
\hline 白术 & Bai Zhu & Atractylodes macrocephala Koidz. & Atractylodis Macrocephalae Rhizoma & Dry rhizoma & 12 \\
\hline 获苓 & Fu Ling & Poria cocos (Schw.) Wolf. & Indian Bread Poria & Dry rhizoma & 15 \\
\hline 淫羊蕉 & Yin Yang Huo & Epimedium brevicornu Maxim. & Epimedii Folium & Herba & 15 \\
\hline 葫芦巴 & $\mathrm{Hu}$ Lu Ba & Trigonella foenum-graecum $\mathrm{L}$. & $\begin{array}{l}\text { Common Fenugreek Seed Semen } \\
\text { Trigonellae }\end{array}$ & Dry seed & 15 \\
\hline 补骨脂 & Bu Gu Zhi & Psoralea corylifolia L. & Fructus Psoraleae & Fruit & 12 \\
\hline \multicolumn{6}{|c|}{ For Yin syndrome deficiency, add Yangyin formula: } \\
\hline $\begin{array}{l}\text { Chinese } \\
\text { name }\end{array}$ & $\begin{array}{l}\text { Chinese } \\
\text { name(Pinyin) }\end{array}$ & Pharmaceutical name & English name & $\begin{array}{l}\text { Produced } \\
\text { from }\end{array}$ & $\begin{array}{l}\text { Dosage } \\
\text { (grams) }\end{array}$ \\
\hline 北沙参 & Bei Sha Shen & Glehnia littoralis (A.Gray) F.Schmidt ex Miq. & Coastal Glehnia Root & Dry rhizoma & 30 \\
\hline 南沙参 & Nan Sha Shen & Adenophora stricta Miq. & Fourleaf Ladybell Root & Dry rhizoma & 30 \\
\hline 天冬 & Tian Dong & Asparagus cochinchinensis (Lour.) Merr. & Cochinchinese Asparagus Root & Dry rhizoma & 15 \\
\hline 麦冬 & Mai Dong & Ophiopogon japonicus (Thunb.) Ker Gawl. & Dwarf Lilyturf Tuber & Dry rhizoma & 15 \\
\hline 百合 & Bai He & Lilium brownii var. viridulum Baker & Lilii Bulbus & Scale leaf & 15 \\
\hline 女贞子 & Nv Zhen Zi & Ligustrum lucidum W.T.Aiton & Fructus Ligustri Lucidi & Fruit & 12 \\
\hline
\end{tabular}

baseline analysis and analysis on enumeration data, and rank-sum test was utilized for analysis on ordered data (NCI-CTC classification of AEs). The DFS time referred to the time interval between the date of enrolment and the date of disease recurrence or metastasis, the data were analyzed with log-rank test, the DFS and OS curves were estimated using the Kaplan-Meier method, the $95 \%$ confidence interval (CI) was calculated using the Greenwood formula, and COX regression analysis was employed for stratified analysis. All of the data were eligible random data obtained from patients in the full-analysis set.

\section{Results}

From February 17th, 2012 to March 20th, 2015, a total of 233 patients with stage IB-IIIA lung adenocarcinoma after radical surgery were selected from 7 hospitals. Among them, 116 patients were randomly assigned into the ACT + CHMF group, and 117 patients were assigned into the $\mathrm{ACT}+$ placebo group. In each group, there were patients 


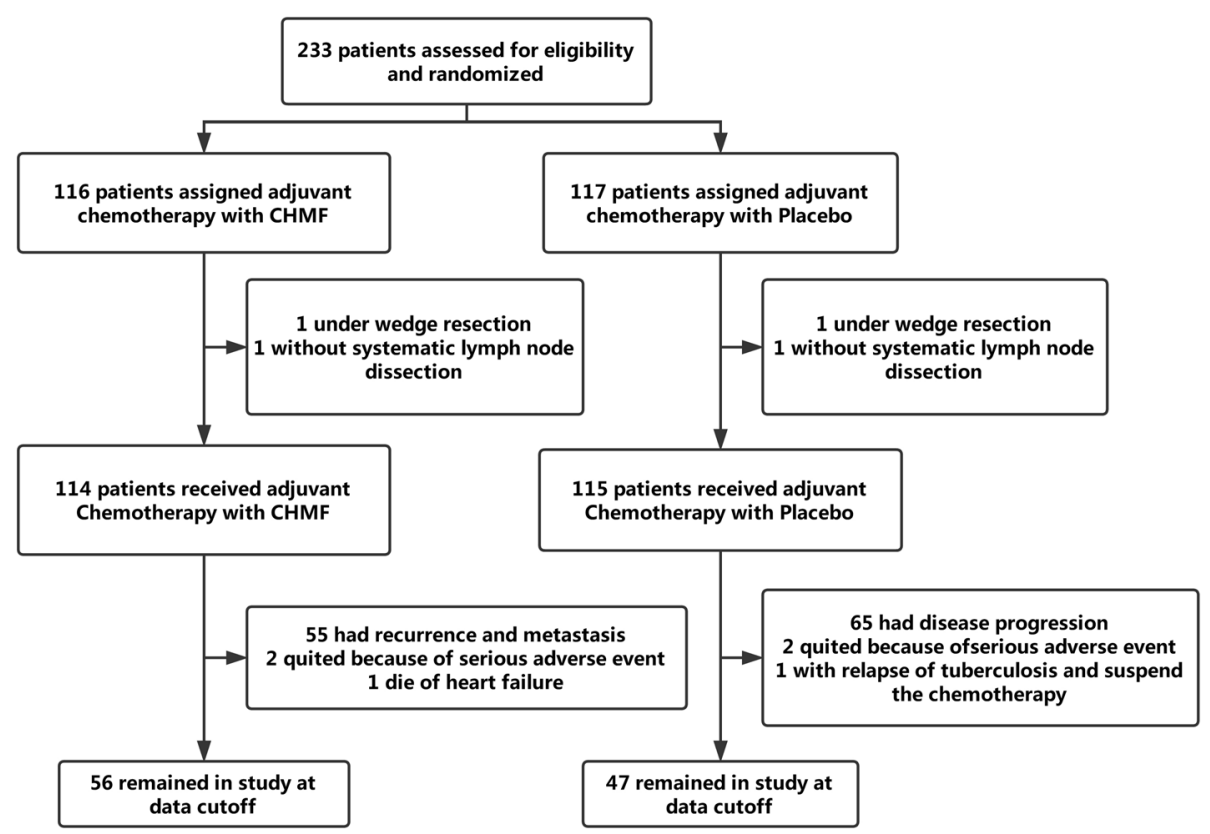

Fig. 1 Study profile. Data cutoff was April 16, 2018. CHMF, Chinese herbal medicine formula

excluded from analysis because of the performance of wedge resection and without systematic mediastinal lymphadenectomy. At the time of data cutoff (April 16th, 2018), a total of 55 patients experienced recurrence and metastasis in the chemotherapy + CHMF group. Two patients experienced serious side effects and withdrew from study and one patient died from heart failure. In the chemotherapy + placebo group, a total of 65 patients experienced recurrence and metastasis, two patients withdrew because of serious side effects and one patient discontinued chemotherapy for relapse of tuberculosis. These patients were included in the intention-to-treat efficacy analysis as shown in Fig. 1. There was no significant difference in age, sex, Eastern Cooperative Oncology Group (ECOG) Performance Status, smoking history, resection procedure, video assistance or not, p-TNM stage, chemotherapy regimens and TCM syndrome between two groups. See Table 2.

Effect of CHMF Addition to ACT on Toxicity and Side Effects Adverse events including toxicity and side effects were reported according to CTC AE issued by NCI (https:// ctep.cancer.gov/protocolDevelopment/Electronicapplica tions/docs/ctcaev3.pdf). The five most common side effects caused by regimens occurring in $>40 \%$ of patients were fatigue, loss of appetite, nausea, leucopenia and vomiting. At 1 week after last course of treatment, the incidence of these side effects decreased in the CHMF group. The incidence of grade 3-4 serious adverse events (SAEs) was27.2\% (31/114) and 30.4\% (35/115) respectively in the CHMF group and the placebo group respectively. See Table 3. In addition, the incidence of dry mouth, diarrhea and thrombocytopenia decreased significantly in the CHMF group compared with the placebo group, with $24.6 \%$ vs. $39.1 \%$ ( 1 week after chemotherapy at cycle $2, P=0.017$ ), $7.9 \%$ vs. $17.4 \%$ ( 1 week after chemotherapy at cycle $3, P=0.033)$ and $5.3 \%$ vs. $13 \%$ ( 1 week after chemotherapy at cycle $4, P=0.019$ ) in the two groups respectively. Also, at 2 weeks after chemotherapy at cycle 4 , fatigue and diarrhea were significantly reduced in the CHMF group than in the placebo group $(P=0.028,0.025$, respectively). See Fig. 2 .

\section{Effect of CHMF Addition to ACT on the DFS}

The secondary endpoint of the study was DFS, defined as the time interval between the date of enrolment and the date of disease recurrence or metastasis. After a median follow-up of 53.6 months (IQR 5.6-75.1). Kaplan-Meier survival analysis showed a median DFS time of 51.5 and 37.1 months in the CHMF group and the placebo group respectively $[\mathrm{HR}=0.77(0.54-1.11)$, $P=0.16]$. Subgroup analysis was conducted by TNM stage. For patients with disease at stage II to IIIa, there was no significant difference in DFS between the groups. However, in patients with stage IB lung adenocarcinoma, Kaplan-Meier survival analysis showed a significant effect of ACT plus CHMF on DFS [HR $=0.53(0.28-0.99)$, $P=0.046]$. See Fig. 3 A1-4.

\section{Effect of CHMF Addition to ACT on the OS}

OS was defined as the time interval between the date of enrolment and the date of death by any cause. Kaplan- 
Table 2 Baseline characteristics and demographics of the population received adjuvant treatment. Data are expressed as numbers (percentage) or mean (range)

\begin{tabular}{|c|c|c|c|}
\hline & $\begin{array}{l}\text { Adjuvant Chemo + CHMF } \\
(n=114)\end{array}$ & $\begin{array}{l}\text { Adjuvant Chemo + Placebo } \\
(n=115)\end{array}$ & $\begin{array}{l}P_{-} \\
\text {value }\end{array}$ \\
\hline Gender, n (\%) & & & 0.596 \\
\hline Male & $57(50 \%)$ & $61(53 \%)$ & \\
\hline Female & $57(50 \%)$ & $54(47 \%)$ & \\
\hline Age & $59.4 \pm 7.4$ & $59.6 \pm 7.5$ & 0.861 \\
\hline ECOG PS, n (\%) & & & 0.99 \\
\hline 0 & $2(1.8 \%)$ & $2(1.7 \%)$ & \\
\hline 1 & $111(97.4 \%)$ & $112(97.4 \%)$ & \\
\hline 2 & $1(0.8 \%)$ & $1(0.9 \%)$ & \\
\hline Smoking history, n (\%) & & & 0.224 \\
\hline Smoked & $40(35.1 \%)$ & $50(43.5 \%)$ & \\
\hline Never smoke & $74(64.9 \%)$ & $65(56.5 \%)$ & \\
\hline Resection procedure, n (\%) & & & 0.333 \\
\hline Lobectomy & $108(94.7 \%)$ & $112(97.4 \%)$ & \\
\hline Sublobectomy & $6(5.3 \%)$ & $3(2.6 \%)$ & \\
\hline Video-assaciated, n (\%) & & & 0.689 \\
\hline Yes & $64(56.1 \%)$ & $68(59.1 \%)$ & \\
\hline No & $50(43.9 \%)$ & 47 (40.9\%) & \\
\hline pTNM stage, n (\%) & & & 0.998 \\
\hline $\mathrm{lb}$ & $55(48.2 \%)$ & $56(48.7 \%)$ & \\
\hline$\|$ & $22(19.3 \%)$ & $22(19.1 \%)$ & \\
\hline III & $37(32.5 \%)$ & $37(32.2 \%)$ & \\
\hline Chemo regimens, n (\%) & & & 0.688 \\
\hline NP & $48(42.1 \%)$ & 45 (39.1\%) & \\
\hline NC & $66(57.9 \%)$ & $70(60.9 \%)$ & \\
\hline TCM syndrome, n (\%) & & & 0.862 \\
\hline Qi deficiency & $55(56.1 \%)$ & $58(50.4 \%)$ & \\
\hline Yin deficiency & $10(8.8 \%)$ & $8(7.0 \%)$ & \\
\hline Qi-Yin deficiency & 49 (35.1\%) & 49 (42.6\%) & \\
\hline
\end{tabular}

Abbreviations: Chemo, chemotherapy; CHMF Chinese herbal medicine formula, ECOG PS Eastern Cooperative Oncology Group Performance Status, NP Vinorelbine plus cisplatin, NC Vinorelbine plus Carboplatin

Meier survival analysis did not show a statistically significant improvement in OS in the CHMF group [HR = $0.89(0.49-1.64), P=0.72]$. At the end of the follow-up period, two groups did not reach the median survival time. Subgroup analysis also showed a similar benefit in the groups according to TNM stage. See Fig. 3 B1-4.

\section{Discussion}

According to the NCCN clinical practice guidelines in oncology, cisplatin-based two drugs ACT was given to postsurgical NSCLC patients with resectable stage II IIIA NSCLC or stage IB NSCLC with high-risk factors [6]. To our knowledge, this is the first multicenter, randomized, and double-blind trial to demonstrate the advantages of ACT combined with CHMF in improving DFS.

The ANITA initiated a randomized phase III trial in patients with completely resected stage IB, II, and IIIA NSCLC in 2006, and found a survival benefit for ACT after a median follow-up of 76 months. The analysis showed a median survival of 65.7 months in the chemotherapy group and 43.7 months in the observation group. In our trail, after a median follow-up of 53.6 months, analysis did not show a statistically significant improvement in OS in the CHMF group [HR $=0.89(0.49-1.64)$, $P=0.72]$. The groups did not reach the median survival time, possibly because of the improved selectivity of cancer drugs for patients when recurrence and metastasis 
Table 3 Adverse events during the adjuvant treatment phases. Values are expressed as numbers (percentage). Table presents grade I to IV adverse events in all patients during the adjuvant treatment phases. Adverse events are listed in descending order of frequency in the total patient population

\begin{tabular}{|c|c|c|c|c|c|c|}
\hline & \multicolumn{3}{|c|}{ Adjuvant chemo + CHMF (N = 114) n (\%) } & \multicolumn{3}{|c|}{ Adjuvant chemo+ Placebo $(N=115) n(\%)$} \\
\hline & Grade 1/2 & Grade $3 / 4$ & Total & Grade 1/2 & Grade $3 / 4$ & Total \\
\hline Fatigue & $90(78.9 \%)$ & $1(0.8 \%)$ & $91(79.7 \%)$ & $89(77.45 \%)$ & 0 & $89(77.45 \%)$ \\
\hline Loss of appetite & $88(77.2 \%)$ & 0 & $88(77.2 \%)$ & $94(81.7 \%)$ & 0 & $94(81.7 \%)$ \\
\hline Nausea & $77(67.5 \%)$ & $1(0.8 \%)$ & $78(68.3 \%)$ & $77(67 \%)$ & 0 & 77 (67\%) \\
\hline Leucopenia & 49 (43.6\%) & $8(7 \%)$ & $57(50.6 \%)$ & $40(34.8 \%)$ & $15(13 \%)$ & $55(47.8 \%)$ \\
\hline Vomiting & $43(37.3 \%)$ & $6(4.4 \%)$ & $49(41.7 \%)$ & $47(40.9 \%)$ & $1(0.8 \%)$ & $48(41.7 \%)$ \\
\hline Elevated TB & $37(32.5 \%)$ & $1(0.8 \%)$ & $38(33.3 \%)$ & $40(34.8 \%)$ & 0 & $40(34.8 \%)$ \\
\hline Constipation & $37(32.5 \%)$ & $1(0.8 \%)$ & $38(33.3 \%)$ & 39 (33.9\%) & 0 & 39 (33.9\%) \\
\hline Neutropenia & $34(29.8 \%)$ & $11(9.7 \%)$ & $45(39.5 \%)$ & $37(32.2 \%)$ & $17(14.8 \%)$ & $54(47 \%)$ \\
\hline Anaemia & $29(25.4 \%)$ & 0 & $29(25.4 \%)$ & $28(24.3 \%)$ & $1(0.8 \%)$ & $29(25.1 \%)$ \\
\hline Dry mouth & $28(24.6 \%)$ & 0 & $28(24.6 \%)$ & $45(39.1 \%)$ & 0 & $45(39.1 \%)$ \\
\hline Pain & $28(24.7 \%)$ & 0 & $28(24.7 \%)$ & 37 (32.2\%) & 0 & $37(32.2 \%)$ \\
\hline Alopecia & $13(11.4 \%)$ & 0 & $13(11.4 \%)$ & $14(12.2 \%)$ & 0 & $14(12.2 \%)$ \\
\hline ALT/AST increased & $12(10.5 \%)$ & $1(0.8 \%)$ & $13(11.3 \%)$ & $15(11.3 \%)$ & 0 & $15(11.3 \%)$ \\
\hline Elevated GGT & $11(9.6 \%)$ & $2(1.8 \%)$ & $13(11.4 \%)$ & $9(7.8 \%)$ & $1(0.8 \%)$ & $10(8.6 \%)$ \\
\hline Diarrhoea & $9(7.9 \%)$ & 0 & $9(7.9 \%)$ & $20(17.4 \%)$ & 0 & $20(17.4 \%)$ \\
\hline Thrombocytopaenia & $6(5.3 \%)$ & 0 & $6(5.3 \%)$ & 15 (13\%) & $1(0.8 \%)$ & $16(13.8 \%)$ \\
\hline Elevated CRE & $4(3.5 \%)$ & 0 & $4(3.5 \%)$ & $1(0.9 \%)$ & 0 & $1(0.9 \%)$ \\
\hline Weight-decrease & $4(3.5 \%)$ & 0 & $4(3.5 \%)$ & 7 (6.1\%) & 0 & $7(6.1 \%)$ \\
\hline Rash & $3(2.6 \%)$ & 0 & $3(2.6 \%)$ & $2(1.7 \%)$ & 0 & $2(1.7 \%)$ \\
\hline Arhythmia & $2(1.8 \%)$ & 0 & $2(1.8 \%)$ & $2(1.7 \%)$ & $1(0.8 \%)$ & $3(2.5 \%)$ \\
\hline Pruritus & $2(1.8 \%)$ & 0 & $2(1.8 \%)$ & 4 (3.5\%) & 0 & $4(3.5 \%)$ \\
\hline Elevated ALP & $1(0.8 \%)$ & 0 & $1(0.8 \%)$ & 1 (0.9\%) & 0 & $1(0.9 \%)$ \\
\hline
\end{tabular}
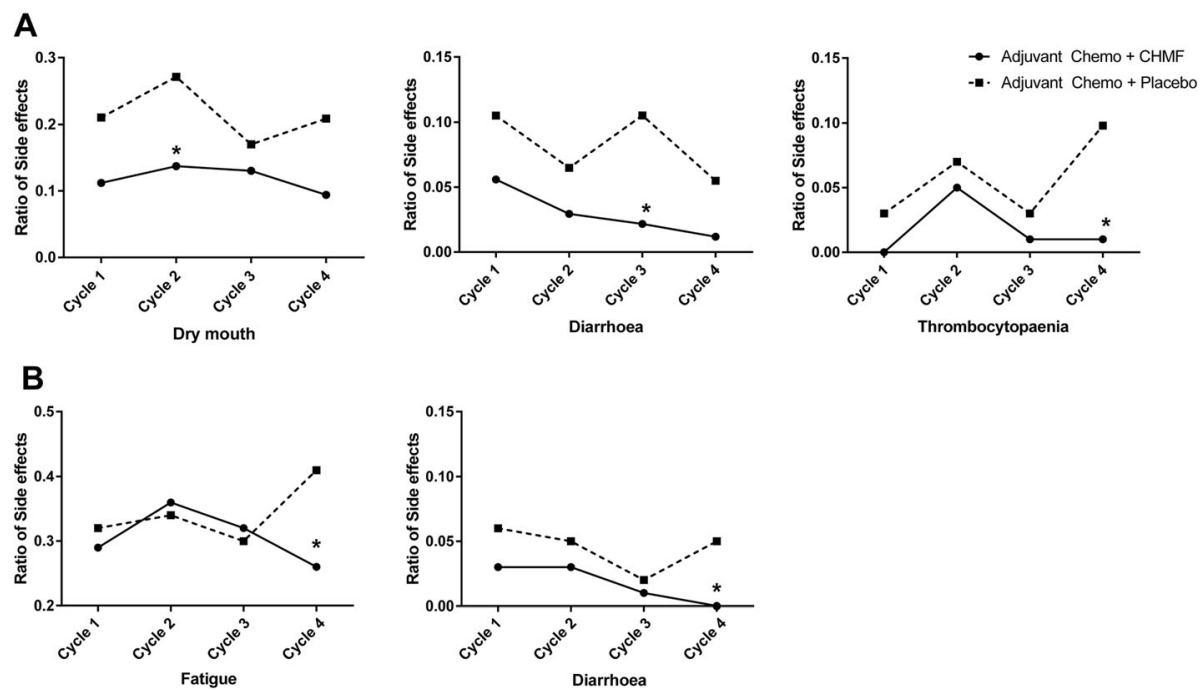

Fig. 2 Figures present adverse events which significantly decreased at one week (a) and two weeks (b) following chemotherapy in the CHMF group (solid line) compared with the placebo group (dotted line). * stands for $P<0.05$ 


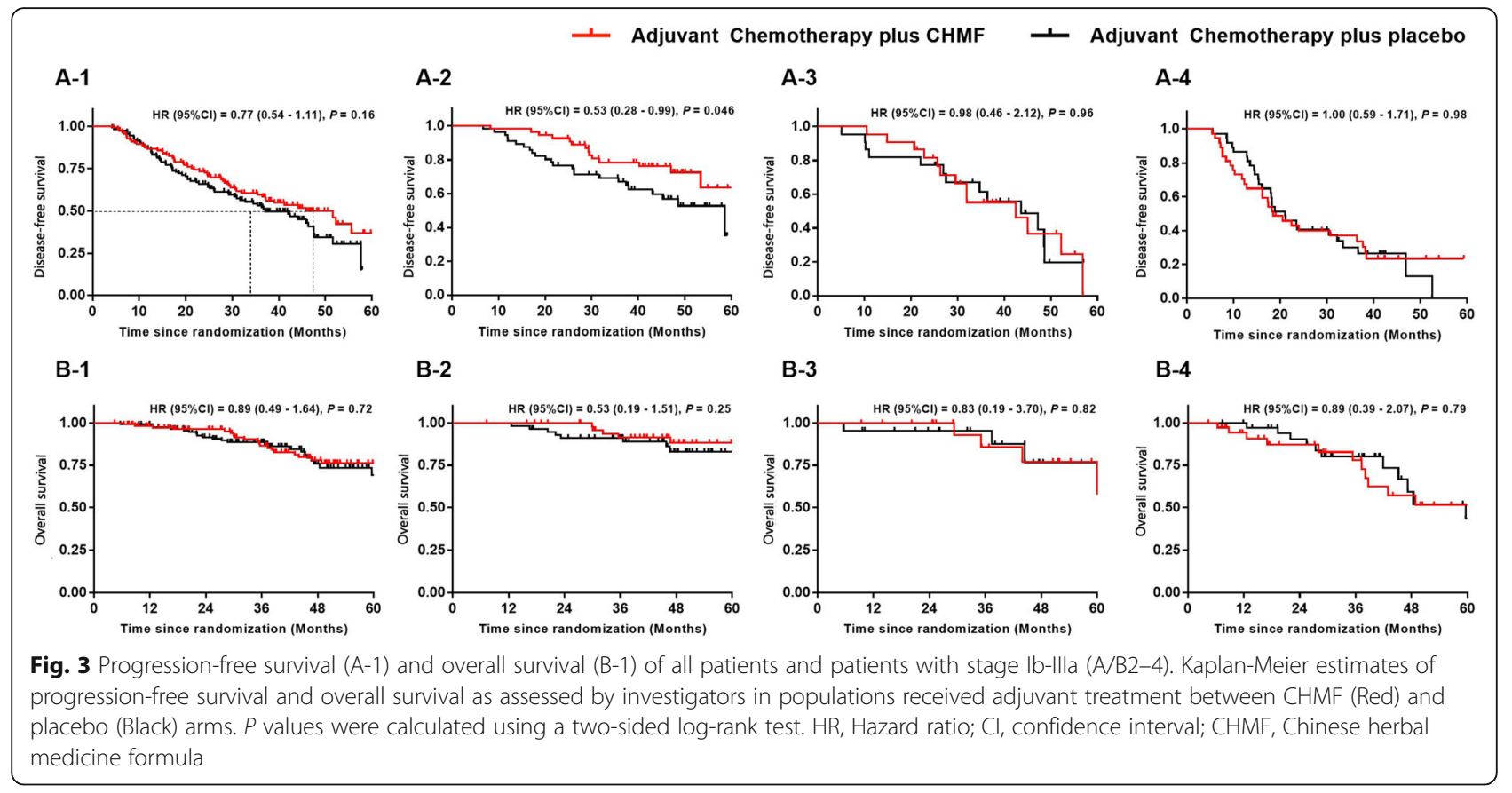

occurred. After chemotherapy, CHMF treatment might also be required in the placebo group and targeted therapies were often given, which were the confounding factors for OS assessment.

In the current study, it was found that there was an improvement of DFS in the CHMF group without statistical significance $[\mathrm{HR}=0.77(0.54-1.11), P=0.16]$. The subset analysis of stage showed that ACT + CHMF was able to obviously prolong the DFS time in patients with stage IB disease $[\mathrm{HR}=0.53(0.28-0.99), P=0.046]$. The DFS time was not significantly different between the groups in patients with stage II and IIIA disease.

According to the 7th TNM classification for lung cancer, the 5-year survival rate of stage IB lung cancer is $66 \%$, significantly lower than that of stage IA cancer (82\%) [2]. Stage IB NSCLC patients with high-risk factors including poor differentiated tumor, tumor size > $4 \mathrm{~cm}$, visceral pleural invasion, vascular invasion and wedge resection [6] are advised to receive ACT based on a prospective randomized clinical trial [16]. In addition to the above factors, pathological classification also has guiding significance for patients to receive ACT or not. Solid/micropapillary patterns were associated with poor prognosis [17]. The predominant micropapillary/solid pattern in patients with stage IB lung adenocarcinoma who received ACT had obviously lower cumulative incidences of lung cancerrelated death and recurrence $[18,19]$. Therefore, CHMF alone or in combination with ACT has potential advantages in patients with stage IB lung adenocarcinoma after radical surgery.
In a previous study, it was shown that chemotherapy could lower the quality of life in a short term, which was mainly associated with fatigue, nausea and vomiting [4]. Adjuvant vinorelbine plus cisplatin had an acceptable level of toxicity and prolonged disease-free survival and overall survival among patients with completely resected NSCLC. Analysis of adverse events indicated that anemia, neutropenia, fatigue, loss of appetite, and vomiting were frequent in the patients with incidence over $50 \%[4,7]$.

Quality of life assessment was performed in 359 patients, and the overall incidence of neutropenia was no more than $50 \%$, with 39.5 and $47 \%$ in the CHMF group and the control group respectively. In this trial, the most frequent side effects caused by NP/NC ACT included fatigue, loss of appetite, nausea, leucopenia and vomiting. The incidence of grade 3-4 toxic and side effects was 27.2 and $30.4 \%$ in the ACT + CHMF group and the $\mathrm{ACT}+$ placebo group respectively. The incidence of grade 3-4 leucopenia and neutropenia was $7 \%$ vs. 13 and $9.7 \%$ vs. $14.8 \%$ respectively, without statistical significance $(P=0.129 ; P=0.236)$. These results were similar to those in patients with stage IB IIIA NSCLC that received NP/NC ACT [4]. In the ANITA trial, the incidence of agranulocytosis was lower than $85 \%$ in the chemotherapy group.

Nevertheless, the incidence of grade 3-4 vomiting was higher in the ACT + CHMF group than in the ACT + placebo group ( $4.4 \%$ vs. $0.8 \%, P=0.053)$, which may be associated with the fact that Chinese medicines were taken orally immediately after chemotherapy and helps to determine the best time to take Chinese medicines. 
Meanwhile, we separately compared the toxic and side effects at 1 and 2 weeks after chemotherapy. In comparison of side effects caused by chemotherapy at 1 week after chemotherapy, dry mouth was more remarkable in the placebo group than in the CHMF group at cycle 2 $(P=0.017)$; diarrhea was more evident in the placebo group than in the CHMF group at cycle $3(P=0.033)$; and thrombocytopenia was more markedly in the placebo group than in the CHMF group at cycle $4(P=0.019)$. At 2 weeks after chemotherapy, fatigue and diarrhea were more obvious in the placebo group than in the $18 \mathrm{MF}$ group at cycle $4(P=0.028, P=0.025)$. This indicated that Chinese medicines alleviated the symptoms (diarrhea, thrombocytopenia and fatigue) mostly at cycles 3 and 4 , consistent with the characteristics of Chinese medicines that they act slowly with sustained effect.

Our previous study revealed that Yin deficiency and Qi deficiency of the lung were the major types of syndrome in lung cancer. Yin deficiency of the lung was constantly present in the disease, more remarkable at the early stage, which was associated with the physiological property of the lung, i.e. preference for moistness and intolerance to dryness [20]. Therefore, treatment nourishing Yin was given in the present study to improve the symptom dry mouth after chemotherapy. Meanwhile, progress-free survival (PFS) was analyzed in patients with different types of syndrome, and no difference was observed in prognosis. In early-stage lung adenocarcinoma, intervention with Chinese medicines + $\mathrm{ACT}$ was more effective in regulating imbalance of Yin and Yang and improving the symptoms, hence playing a synergistic role in fighting against tumor.

The limitation of this study lies in the fact that different prescriptions were used for different types of syndrome and each of the prescriptions was comprised of multiple ingredients, demanding further study.

\section{Conclusion}

In conclusion, Chinese herbal medicines enable ACT to prolong the DFS time in patients with stage IB lung adenocarcinoma, and reduce the toxic and side effects caused by chemotherapy, including dry mouth, fatigue, diarrhea and thrombocytopenia.

\footnotetext{
Abbreviations

ACT: Adjuvant chemotherapy; ANITA: Adjuvant Navelbine International Trialist Association; ASCO: American Society of Clinical Oncology; CHMFs: Chinese herbal medicine formulas; Cl: Confidence interval; CRO: Clinical research organization; DFS: Disease-free survival; ECOG: Eastern Cooperative Oncology Group; GCP: Good Clinical Practice; GMP: Good Manufacturing Practice; IRBs: Institutional review boards; LAC: Lung adenocarcinoma; LACE: Lung Adjuvant Cisplatin Evaluation collaborative group; NCCN: National Comprehensive Cancer Network; NCl: National Cancer Institute; NSCLC: Nonsmall cell lung cancers; OS: Overall survival; PS: Performance status; QoL: Quality of life; SAEs: Serious adverse events; TCM: Traditional Chinese medicine
}

\section{Acknowledgements}

The authors would like to thank Prof. Yu Jin and Jianfeng Cai from East China University of Science and Technology, for their technical assistance and instructions.

\section{Authors' Contributions}

YBG, LX and LJ conceived and designed the experiments. DZ, JLY, JQL, LYW, ZWC, YJJ, ZWZ, WSS, WRZ, JFX and YG performed the experiments and QW, SFW wrote the paper. QW, SFW and PQC collected and analyzed the data. LJ and PQC provided technical expertise. YBG and LX provide assistance with revising this manuscript. All authors read and approved the manuscript.

\section{Funding}

This work was supported by Shanghai Municipality Science and Technology Commission Foundation Key Project (16401970700, ZYBZ-2017009, 11DZ1973200), Shanghai Hospital Development center Foundation (16CR1036B). Shanghai Science and Technology Innovation Project of Traditional Chinese Medicine (19401934400,ZYKC 201601020), China Association of Chinese Medicine (CACM-2017- QNRC2-C15), Special Scientific Research for Traditional Chinese Medicine (201307006), Shanghai Wu Mengchao Medical Technology Foundation (LCYX 20160705-001), Shanghai Three-year Action Plan to Further Accelerate the Development of Traditional Chinese Medicine (ZY (2018-2020)-FWTX-6009), and National Natural Science Foundation of China $(81704035,81803888)$

\section{Availability of Data and Materials}

To support the results reported, data and materials can be found on the website https://www.clinicaltrials.gov/ct2/show/NCT01441752?term=01441 752\&rank=1

\section{Ethics Approval and Consent to Participate}

The study protocol was approved by the institutional review boards (IRBs) of the participating medical centers, the independent National Ethics Committee, the Chinese Medicinal Agency, and was registered under the identifier NCT01441752 at the Clinicaltrials.Gov website. The study was performed in accordance with the Declaration of Helsinki.

\section{Consent for Publication}

Not applicable.

\section{Competing Interests}

The authors declared that they have no competing interests.

\section{Author details}

'Department of Oncology, Yueyang Hospital of Integrated Traditional Chinese and Western Medicine, Shanghai University of Traditional Chinese Medicine, Shanghai, China. ${ }^{2}$ Institute of Clinical Immunology, Yueyang Hospital of Integrated Traditional Chinese and Western Medicine, Shanghai University of Traditional Chinese Medicine, Shanghai, China. ${ }^{3}$ Department of Thoracic Surgery, Fudan University Shanghai Cancer Center, Shanghai, China. ${ }^{4}$ Lung Tumor Clinical Medical Center, Shanghai Chest Hospital, Shanghai Jiaotong University, Shanghai, China. ${ }^{5}$ Department of Oncology, First Hospital Affiliated to Tianjin College of Traditional Chinese Medicine, Tianjin, China. ${ }^{6}$ Department of Oncology, Changshu No.2 People's Hospital, Jiangsu, China. ${ }^{7}$ Department of Oncology, Jiangyin People's Hospital, Jiangsu, China. ${ }^{8}$ Department of Traditional Chinese Medicine, Ruijin Hospital Affiliated to Shanghai Jiaotong University School of Medicine, Shanghai, China.

${ }^{9}$ Department of Oncology, Shanghai Pulmonary Hospital, Tongji University School of Medicine, Shanghai, China. ${ }^{10}$ Department of Oncology, Shanghai East Hospital, Tongji University School of Medicine, Shanghai, China.

Received: 31 October 2019 Accepted: 4 February 2020

Published online: 01 March 2020

\section{References}

1. Siegel RL. Cancer statistics. CA Cancer J Clin. 2018;68:7-30.

2. Goldstraw P, Chansky K, Crowley J, et al. The IASLC lung cancer staging project: proposals for revision of the TNM stage groupings in the forthcoming (eighth) edition of the TNM classification for lung cancer. J Thorac Oncol. 2016;11(1):39-51. 
3. Pignon JP, Tribodet H, Scagliotti GV, et al. Lung adjuvant cisplatin evaluation: a pooled analysis by the LACE collaborative group. J Clin Oncol. 2008;26:3552.

4. Douillard JY, Rosell $R$, De Lena M, et al. Adjuvant vinorelbine plus cisplatin versus observation in patients with completely resected stage IB-IIIA nonsmall-cell lung cancer (adjuvant Navelbine international Trialist association [ANITA]): a randomized controlled trial. Lancet Oncol. 2006;7:719-27.

5. Pisters KM, Evans WK, Azzoli CG, et al. Cancer Care Ontario and American Society of Clinical Oncology adjuvant chemotherapy and adjuvant radiation therapy for stages I-IIIA resectable non-small-cell lung cancer guideline. J Clin Oncol. 2007:25:5506

6. National Comprehensive Cancer Network (NCCN). NCCN Clinical practice guidelines in oncology. lung Cancer (Version 3.2011)[DB/OL]. http://www. ncen.org.

7. Winton $T$, Livingston $R$, Johnson $D$, et al. Vinorelbine plus cisplatin vs observation in resected non-small-cell lung cancer. N Engl J Med. 2005;352: 2589-97.

8. Wang J, Chen YL, Ping C. Clinical observations on treating 40 cases of locally advanced non-small-cell lung cancer with the Zilongjin tablet. Chin J Trad Chin Med. 2016;8:15-7.

9. Liang J, Zhang L. Survival effect of Rongyan capsule, a maintenance treatment for advanced non-small-cell lung cancer. Sichuan Zhong Yi. 2013;1:100-1.

10. Zhao X, Dai X, Wang S. Traditional Chinese medicine integrated with chemotherapy for stage II-IIIA patients with non-small-cell lung cancer after radical surgery: a retrospective clinical analysis with small sample size. Evid Based Complement Alternat Med. 2018:10:1155.

11. Jiao L, Dong C, Liu J, et al. Effects of Chinese medicine as adjunct medication for adjuvant chemotherapy treatments of non-small cell lung cancer patients. Sci Rep. 2017;7:46524.

12. Wang $Q$, Jiao $L$, Wang $S$, et al. Maintenance chemotherapy with Chinese herb medicine formulas vs. with placebo in patients with advanced nonsmall cell lung cancer after first-line chemotherapy: a multicenter, randomized, double-blind trial. Front Pharmacol. 2018:9:1233.

13. Nakaya S. Question about clinical trial of traditional Chinese medicine. Prog Japanese Med. 2003;24(2):91-3.

14. Wang R, Luo D, He Z, et al. Objective evaluation of Huoxiang Zhengq particles placebo. Chin J Exp Tradit Med Formulae. 2014;20(12):91-5.

15. Scagliotti GV, Pastorino U, Johan F, et al. Randomized phase III study of surgery alone or surgery plus preoperative cisplatin and gemcitabine in stages IB to IIIA non-small-cell lung cancer. J Clin Oncol. 2012;30(2):172-8.

16. Strauss GM, Herndon JE, Maddaus MA, et al. Adjuvant paclitaxel plus carboplatin compared with observation in stage IB non-small-cell lung cancer: CALGB 9633 with the cancer and leukemia group B, radiation therapy oncology group, and north central cancer treatment group study groups. J Clin Oncol. 2008;26:5043-51.

17. Tsao MS, Marguet S, Le Teuff G, et al. Subtype classification of lung adenocarcinoma predicts benefit from adjuvant chemotherapy in patients undergoing complete resection. J Clin Oncol. 2015;58:8335

18. Ma M, She Y, Ren Y, et al. Micropapillary or solid pattern predicts recurrence free survival benefit from adjuvant chemotherapy in patients with stage IB lung adenocarcinoma. J Thorac Dis. 2018;10(9):5384-93.

19. Qian F, Yang W, Wang R, et al. Prognostic significance and adjuvant chemotherapy survival benefits of a solid or micropapillary pattern in patients with resected stage IB lung adenocarcinoma. J Thorac Cardiovasc Surg. 2017;50022-5223(17):32433-9.

20. Fu YL, Tu HB, Dong ZY, et al. Correlation of TCM syndrome types, TNM staging and tumor antigen in lung cancer. World Sci Technol Modern Trad Chin Med. 2015;17(6):1210-4

\section{Publisher's Note}

Springer Nature remains neutral with regard to jurisdictional claims in published maps and institutional affiliations.

Ready to submit your research? Choose BMC and benefit from:

- fast, convenient online submission

- thorough peer review by experienced researchers in your field

- rapid publication on acceptance

- support for research data, including large and complex data types

- gold Open Access which fosters wider collaboration and increased citations

- maximum visibility for your research: over $100 \mathrm{M}$ website views per year

At BMC, research is always in progress.

Learn more biomedcentral.com/submissions 